\title{
TTR
}

Traduction, terminologie, re?daction

\section{Les Projets d' illustration " de la langue vernaculaire et leurs héritages littéraires}

\section{Mawy Bouchard}

Volume 9, numéro 2, 2e semestre 1996

Parcours de traduction

Pathways of Translation

URI : https://id.erudit.org/iderudit/037258ar

DOI : https://doi.org/10.7202/037258ar

Aller au sommaire du numéro

\section{Éditeur(s)}

Association canadienne de traductologie

ISSN

0835-8443 (imprimé)

1708-2188 (numérique)

Découvrir la revue

Citer cet article

Bouchard, M. (1996). Les Projets d'« illustration » de la langue vernaculaire et leurs héritages littéraires. TTR, 9(2), 47-74. https://doi.org/10.7202/037258ar
Résumé de l'article

RÉSUMÉ : Les Projets d"' illustration " de la langue vernaculaire et leurs héritages littéraires - Dante écrivit, peu après Vita nova, en 1305, son De Vulgari eloquentia, texte qui servit de " manifeste ", d'abord aux champions des vernaculaires italiens, ensuite aux défenseurs des langues vulgaires européennes en pleine expansion dès la fin du XIVe siècle. Dante suggérait aux poètes désireux d'" illustrer " leur langue maternelle de composer une oeuvre aussi magnifique que celle de son grand maître romain, Virgile. Une oeuvre sans dénomination générique, mais définie par son vers hendécasyllabe et ses matières " illustres " : salus, virtus, venus. Boccace fut le premier disciple de Dante. Il répondit à l'appel de son maître, en écrivant une oeuvre dédiée à Mars, le tout-puissant dieu guerrier, seul capable d'accorder la gloire éternelle aux pauvres mortels. La Teseida délie nozze d1 Emilia servit de modèle, quelques années seulement après sa parution, aux poètes anglais et français. Chaucer traduisit la Teseida, qui devint " The Knight's Tale ", et l'intégra à ses Canterbury Tales vers 1380. En France, un traducteur anonyme du roi René d'Anjou traduisit à son tour, vers 1460, l'oeuvre épique de Boccace, qu'il intitula Thezeo. Au XVIe siècle, époque déterminante dans l'histoire de la langue française, Anne de Graville " translata de vieil langage en nouveau " le Thezeo qu'elle intitula: le Beau romant des deux amans Palamon etArcita et de la belle et saige Emilia. Cette étude suit le parcours de la Teseida de Boccace d'une langue à une autre, en s'intéressant au projet et au " contre-mouvement" de l'illustration des langues vernaculaires européennes esquissé par le grand poète toscan, Dante.
Tous droits réservés @ C TTR: traduction, terminologie, rédaction — Les auteurs, 1996 ce document est protégé par la loi sur le droit d'auteur. L’utilisation des services d'Érudit (y compris la reproduction) est assujettie à sa politique d'utilisation que vous pouvez consulter en ligne.

https://apropos.erudit.org/fr/usagers/politique-dutilisation/ 


\title{
Les Projets d'« illustration » de la langue vernaculaire et leurs héritages littéraires
}

\section{Mawy Bouchard}

\begin{abstract}
" Ce modèle, pour la langue vulgaire, je ne crois pas qu'il puisse etre autre que Pétrarque et Boccace, et celui qui s'éloigne de ces deux-là va à tatons, comme quelqu'un qui chemine dans les ténèbres sans lumières et à cause de cela se trompe souvent de chemin. " Castiglione, le Livre du courtisan.
\end{abstract}

Castiglione et les prétendants au titre de parfait courtisan entonnèrent, au début du XVI' siècle, un air victorieux en l'honneur de la langue vernaculaire italienne. L'auteur du Livre du courtisan exhibait en effet une assurance nouvelle envers la langue vulgaire, assurance que Dante lui-même lui eât enviée. Loin de partager la timide réserve de Boccace à l'égard de sa langue maternelle', Castiglione (1991) reprochait même à celui-ci de ne pas avoir tiré davantage de fierté de ses ceuvres composées en langue vulgaire. Le maître courtisan écrivait à ce propos : " dans ses propres œuvres son jugement l'a trompé, en ce qu'il a peu estimé celles qui lui ont fait honneur, et fait grand cas de celles qui ne valent rien " (p. 10). Afin

1. On verra en effet que Boccace n'a pas toujours assumé l'originalité de sa pensée. Dans ses techanges épistolaires avec Pétrarque, il croit devoir s'excuser auprès de son ami pour avoir agi avec trop de hardiesse en faveur de la langue vulgaire, alors qu'il était débordant d'une impétueuse jeunesse. 
de comprendre cet ample mouvement qu'est la défense et l'illustration de la langue française, il est essentiel d'avoir clairement à l'esprit les préceptes du premier chantre de la langue vulgaire italienne, Dante. Je mettrai d'abord en lumière la pensée de l'auteur du De vulgari eloquentia et du Convivio, afin d'arriver ensuite a dégager, à travers les préfaces, dédicaces et lettres familières de quelques écrivains italiens, anglais et français, un projet d'《 illustration " de la langue vulgaire. On verra comment Boccace, le premier, reconnut et fit sien le projet de celui qu'il considérait comme son maître et comment il en vint à son tour à inspirer les défenseurs d'autres vernaculaires européens. En parallele, nous pourrons observer de quelle manière, paradoxalement, ce projet a échoué dans le pays de ceux qui affirmèrent les premiers leur volonté d'illustrer le vernaculaire et, au contraire, par quel moyen cette entreprise a.obtenu en France et en Angleterre un succès retentissant.

Avant même la Defense et illustration de Joachim Du Bellay, il y eut de nombreuses revendications en faveur de l'affirmation du parler vernaculaire ; entre autres, celles du Champ fleury (1529) de Geofroy Tory, de l'Exhortation atx Français $(1509)^{2}$ de Claude de Seyssel, du Quadrilogue invectif (1422) d'Alain Chartier et bien sûr, pour ne citer qu'un seul Italien, du $D e$ vulgari eloquentia (1305) de Dante. On ne saurait trop insister sur l'importance de ce traité qui camoufle sous des accents ironiques des idées passibles d'accusations d'hérésie, en provoquant un bouleversement épistémologique par rapport au statut de la langue vulgaire ${ }^{3}$. En effet, la langue maternelle de l'homme médiéval ne relève pas de la même conception du langage que la langue

2. Composé en 1509 , mais publié en 1559.

3. Serge Lusignan (1986) expose clairement cette idée d'un bouleversement du statut épistémologique de la langue vernaculaire. En ce qui a trait à la langue française, il a d'abord observé ce bouleversement dans le contexte des luttes territoriales entre rois français et anglais, au cours du $\mathrm{XIV}^{\circledR}$ sitecle. 
" artificielle », apprise par les clercs. La langue vernaculaire était, à l'époque de Dante, une langue essentiellement orale, sans règle formulée, et s'opposait au latin qu'on appelait " grammaire » - régie par des lois et donc artificielle. Le poète toscan désirait faire profiter la langue vulgaire italienne des attributs de la " grammaire " tout en lui conservant son naturel et sa spontanéité. Ce désir appela inévitablement le combat et provoqua de violents conflits parmi les latinistes qui eussent dô assister, dans l'éventualité de la réalisation du projet dantesque, à la disparition de la langue de l'Église. Le projet de Dante (1965, p. 300) était donc audacieux.

Je dis que l'amour naturel par-dessus tout meut l'amant à trois choses : l'une est de magnifier l'aimé ; l'autre est d'être jaloux d'icelui ; l'autre est de le defendre comme chacun peut voir qu'il advient couramment".

L'amour qu'il ressentait pour sa patrie incita Dante à glorifier le seul bien qu'il avait pu emporter avec lui en exil, la langue de son enfance. Le traité De vulgari eloquentia est l'un des premiers écrits connus de Dante (après la Vita nova); le Convivio a été rédigé à peu près en même temps, entre les années 1304 et 1307. Puisque j'identifie ces textes à un " projet » d'illustration de la langue vulgaire ayant partout en Europe des répercussions encore

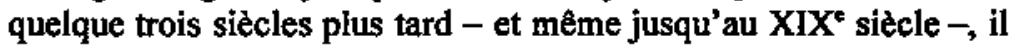
me faut maintenant dégager les grands points d'ancrage de ce qui allait devenir une topique du discours des écrivains, de la fin du Moyen Âge à la Renaissance : la nécessité d' « illustrer 》 sa langue maternelle.

Il convient tout d'abord de bien définir ce que Dante entendait par " parler vulgaire ». Il s'agit, comme on peut le deviner, de la langue commune parlée par les enfants, les femmes et les hommes ; une langue compréhensible par tous les habitants

4. Dorénavant, nous indiquerons les références au $D e$ vulgari eloquentia, « Vulgari ", et au Convivio, « Banquet », placés entre parenthèses, en indiquant le numéro de page correspondant à l'édition de La Pléiade. 
d'une même région, dont notamment les représentants du peuple, mais, il faut le souligner, il n'est que très peu question de celui-ci dans le De vulgari eloquentia de Dante. Le parler vulgaire, c'est tout d'abord la langue des nobles, une langue " que nous parlons sans aucune règle, imitant notre nourrice " (Vulgari, p. 552), et ce par opposition à la langue des savants, le latin. Pour Dante, il y a eu un moment dans l'histoire de l'humanité où chacun pouvait s'exprimer dans une même langue, et ce dans tous les domaines du savoir (Demonet, 1992). C'est par rapport à cet idéal qu'il dénonce la présomption d'une conception du langage qui lui semble hiérarchisée injustement en faveur des représentants de l'Église. Dans une perspective que l'on pourrait qualifier de " gibeline ", Dante plaide la cause des victimes de la tyrannie des Guelfes noirs, défenseurs du Pape :

[...] je dis que l'on peut voir manifestement que le latin aurait a peu de gens donné son bienfait, mais que le vulgaire servira vraiment à beaucoup. Car la bonté de l'esprit, qui attend ce service, est en bien des personnes qui, par mauvais nonchaloir du monde, ont abandonné la littérature à ceux-là qui de dame l'ont faite putain ; et ces nobles esprits sont princes, barons, chevaliers et maints autres nobles gens, non seulement hommes mais femmes, qui sont nombreux et nombreuses à user de cette langue, en personnes vulgaires et non-lettrées. (Banquet, p. 297)

Tout comme Machiavel, qui écrira deux siècles plus tard que le prince doit s'accorder avec son époque, Dante insiste d'abord sur la nécessité, pour la survie d'une langue, de s'adapter au caractère " remuable » et " très changeant » de l'homme. Voilà ce qui se présente comme le premier argument en défaveur du latin : « adonc nulle parlure ne peut être durable et continue, mais comme toutes autres choses humaines, par exemple les us et coutumes, il lui faut changer par écart de lieux et de temps » (Vulgari, p. 567). Ce qui est perçu par tous les hommes savants comme un avantage certain sur la langue vernaculaire, à savoir la pérennité et l'« universalité » du latin, Dante cherche à le montrer comme une inaptitude du langage. De ce point de vue, La langue vulgaire présente cette qualité qu'elle est changeante comme le vent. D'une région, d'un groupe social ou d'une époque à une autre, le parler « naturel » des 
enfants se meut en nuances. Toutefois, il ne faudrait pas se laisser prendre par la rhétorique persuasive de Dante. Son idéal n'est cèrtes pas dans cette mouvance du vernaculaire, mais plutôt dans la spontanéité et la vivacité de la langue toscane vulgaire qui devrait aussi se refléter dans la langue poétique. Tout comme Machiavel, il tentera de régler les problèmes politiques de son pays en proposant un modèle de gouvernement monarchique, unifié par une seule langue « illustre ».: le toscan 5 .

Dante reconnaît trois qualités essentielles à ce vulgaire illustre : il est cardinal, royal et courtois. Par vulgaire « illustre » il entend « quelque chose qui illumine, et qui, illuminé, resplendit » (Vulgari, p. 586). L'autonomie politique du Florentin se reflète ici dans son choix des qualificatifs du vulgaire illustre. Il est d'abord " cardinal ", car il agit en " vrai chef de famille ", il guide l'âme et la parole. Dante explique bien cette notion en offrant à son lecteur l'image de l'huis obéissant au gond. Le choix de ce terme n'apparaitt pas immotivé ; il semble suggérer un système de pouvoir partagé entre la monarchie et l'Église. Mais il faut reconnaître la préférence de Dante pour un gouvernement n'ayant d'autre intérêt que la bonne gestion de son territoire, préférence qui se traduit par le choix des termes " royal » et « courtois ». Le vulgaire illustre est par ailleurs " courtois » en ce sens qu'il obéit à un code et à des règles de convenance. Dante croyait que l'établissement d'une monarchie était le plus sûr moyen de parvenir à l'unification de la langue et du pays. La langue vulgaire illustre ne devait être autre que la langue palatine. Bien qu'aucune monarchie n'eût réussi, à l'époque de Dante, à s'implanter définitivement en Italie, tous les espoirs étaient malgré tout encore permis.

5. Ce projet politique de Dante provoqua l'enthousiasme de ses compatriotes tout au long du XIV" s., mais peu à peu, dés l'époque de Pétrarque, les idées de Dante furent anéanties. Il fallut attendre jusqu'au XIX ${ }^{*}$ s. romantique pour que le projet de Dante suscite à nouveau l'intérêt des écrivains et des politiques. Toutefois, on ne parvint jamais à l'unification linguistique tant souhaitée par Dante. 
Cette première partie de la réflexion de Dante sur les conjonctures politiques et linguistiques de la langue vernaculaire est sans précédent et sans successeur immédiat (Lusignan, 1986, p. 187). Ses répercussions seront ressenties d'abord en Italie mais elles auront plus d'impact en Angleterre et en France. Le Livre II du De vulgari eloquentia offre aux nouveaux champions du vernaculaire un premier modèle thérique. Dante détermine tout d'abord les trois matières dignes du " vulgaire illustre " : « salut, amour, vertu ». Ces trois catégories de sujets correspondent aux trois « puissances » de l'âme humaine, la végétative, l'animale et la rationnelle ${ }^{6}$. Nous verrons que ces trois " genres » distingués par Dante peuvent avoir donné lieu a différentes interprétations et que l'cuvre " totale ", l'cuvre la plus parfaite, a pu être conçue dans une perspective englobant les trois grandes « fins ». À matière illustre doit ensuite correspondre une forme magnifique : la chanson, qui représente, pour le poette toscan, toutes paroles mises en vers ; par " chanson ", il faut entendre "long poème ", comme l'Odyssée, l'lliade, l'Énéide, etc. La chanson, nous dit Dante, doit être considérée supérieure à toutes les autres formes, car elle est en quelque sorte " autosuffisante ": elle ne requiert la participation d'aucune autre forme d'expression - telle la participation de « danseurs qui frappent le sol " pour la ballade. "Il s'ensuit que la chanson doit être estimée plus noble que la ballade; et que par conséquent sa forme

6. Il est intéressant de voir comment ces mêmes préceptes de Dante ont été interprétés quelque cinq siècles plus tard par Victor Hugo. En effet, Hugo élabora, à partir de ses trois puissances de l'ame, une theorie du drame romantique qu'il explicite dans sa preface à Ruy Blas. Il ecrit : " Trois espèces de spectateurs composent ce qu'on est convenu d'appeler le public : premièrement les femmes ; deuxièmement, les penseurs ; troisièmement, la foule proprement dite. Ce que la foule demande presque exclusivement à l'ceuvre dramatique, c'est de l'action [prouesses d'armes] ; ce que les femmes y veulent avant tout, c'est de la passion [amour] ; ce qu'y cherchent plus specialement les penseurs, ce sont des caractères [portraits moraux]. " Il s'agit d'un échange fructueux entre deux grands poètes voulant tous les deux rejoindre le plus vaste auditoire possible. 
est la plus noble de toutes, nul ne doutant que la ballade même l'emporte en noblesse sur le sonnet. " (Vulgari, p. 589) Le style tragique, c'est-à-dire l'équivalent du style sublime en rhétorique, est le plus digne du vulgaire illustre, car il se prête aussi bien aux récits d'actions héro'ques qu'à l'expression de grandes passions (Vulgari, pp. 601-602). Enfin, l'œuvre épique illustre requiert le vers hendécasyllabique - l'équivalent du vers décasyllabique, le vers hérolque français (Vulgari, p. 603).

Le projet de Dante est marqué par l'admiration que le poète vouait à son " maître " romain, Virgile, et par la volonté d'imiter l'initiative " patriotique " des plus grands qui l'ont précédé. Bien que Dante ait aussi promulgué la dignité du genre amoureux et moral, les poètes qui lui ont succédé se sont plutôt évertués à imiter ou surpasser leurs modeles grec, romain et florentin, en chantant les prouesses d'armes de héros mythiques. Le premier poète à reconnaître la grandeur de Dante fut son compatriote Boccace. Il a tout au long de sa vie défendu ce projet qu'il considérait, selon le moment, louable ou " pardonnable ».

\section{$* * *$}

[...] questi fu quel Dante, il quale primo doveva al ritomo delle muse sbandite d'Italia, aprir la via. Per costui la chiarezza del fiorentiono idioma de dimostrata; per costui ogni bellezza del volgar parlare sotto debiti numeri è regolata $[\ldots]$.

Alors qu'il n'avait encore que huit ans, Boccace vit la légende dantesque se composer sous ses yeux. En 1321, Dante s'éteint ; Boccace s'avive. La renommée du grand poète toscan court par l'Italie. Dante laissait un héritage grandiose à tous les amoureux de leur langue " naturelle » : la Commedia. Celle-ci servit autant de modèle que de source d'inspiration à différentes entreprises d'écriture en langue vulgaire. Ainsi la Teseida delle nozze d'Emilia

7. Boccace, Vita di Dante (1888, pp. 10-11). Il n'existe pas de traduction française de la Vita di Dante. 
fut écrite dès les premières années de la carrière poétique de Boccace, vers 1340. La composition de cette ouvre procède du désir de Boccace de remplir le « poste vacant » de poète illustre en langue vulgaire dans le genre épique :

Poi che le Muse nude cominciaro nel cospetto degli uomini ad andare, gia fur di quelli i quaj l'esercitaro con bello stilo in onesto parlare, e altri in amoroso l'operaro; ma tu, o libro, primo a lor cantare di Marte fai gli affanni sostenuti, nel volgar lazio pì̀ mai non veduti. (1992, XII, 84, p. 419)

En répondant à la requête poétique de Dante, Boccace homologuait, en quelque sorte, l'entreprise linguistique et politique de son déjà célèbre prédécesseur et offrait un " prototype " du vulgaire illustre. En outre, il accordait, pour la première fois dans l'histoire littéraire de la langue vernaculaire italienne, le statut d' " auctoritas 》 d un contemporain ne s'exprimant pas en latin. Boccace a ainsi déclenché le début des travaux de translatio studii - au sens de " traduction » - du latin au vernaculaire toscan, et ensuite de l'italien au français et à l'anglais, en France et en Angleterre. La péninsule italique, " centre du monde " aux $\mathrm{XIV}^{*}$ et $\mathrm{XV}^{\circ}$ siècles, n'eut pas de difficulté à entraîner, dans son mouvement de " vulgarisation » de la poésie, tous les pays d'Europe à la recherche d'identités nationales.

Boccace a donc, dans sa jeunesse, poursuivi la gloire que lui promettait la muse Calliope. Il s'est efforcé de reproduire, dans sa langue maternelle, la grandeur et l'élégance des langues grecque et latine. Pour son ouvre, it puisa ainsi son inspiration chez les Anciens, grecs et romains. La Teseida delle nozze d'Emilia ressemble par sa structure et son sujet à la Thébaide de Stace (poète latin ayant vécu entre les années 40 et 96), ainsi qu'au Roman de Thèbes (d'un auteur anonyme du XII siècle). Tout comme les grandes épopées antiques, la Teseida veut consacrer les exploits d'un grand personnage, en relatant rétrospectivement les prouesses d'un 
héros. Boccace exprime dans le premier Livre de son épopée ce désir d' "illustrer " la langue vulgaire dans la seule matière qui, selon lui, n'eût pas son " canon ", à savoir le genre consacré au dieu Mars :

Siate presenti, o Marte rubicondo
nelle tue armi rigido e feroce,
e tu, madre d'Amor, col tuo giocondo
e lieto aspetto, e 'l tuo figliuol veloce
co' dardi suoi possenti in ogni mondo ;
e sostenete e la mano e la voce
di me che' ntendo i vostri effetti dire
con poco bene e pien d'assai martire. (1992, I, 3, p. 12)

La disposition de l'œuvre de Boccace se compare à plusieurs égards à l'Énéide de Virgile : la Teseida est divisée en douze livres où le vers hendécasyllabique préconisé par Dante est roi. Certains thèmes et motifs typiques de l'épopée antique sont employés tels quels ou remaniés par Boccace. Par exemple, les périphrases servant habituellement à décrire les repères temporels sont reprises intégralement par l'auteur ${ }^{8}$, les scènes de bataille sont nombreuses, mais elles se voient souvent transformées allégoriquement. Boccace privilégie aussi les métaphores amoureuses, prêtant au guerrier les attributs de l'amant à la poursuite de sa bien-aimée. Cet alliage du guerrier et de l'amoureux en a embarrassé plusieurs. Thomas $\mathbf{G}$. Bergin (1981), notamment, écrit que la « Teseida [...] is a hybrid, no true epic at all with pretentious epic trappings that strain and distort the simple love romance that it might have been " (p. 118). Et plus loin : " Even more acceptant and colorless, to be sure, is Emilia, the focus of the rivals' desires. There is assuredly no epic

8. Par exemple, au début du Livre II, Boccace veut évoquer le printemps par cette périphrase " omée " : "Nel dolce tempo che il ciel fa belle / le valli e' monti d'erbette e di fiori, / e le piante riveste di novelle / frondi, sopra le quali i loro amori cantan gli uccelli, e le gaie donzelle / di Citerea più senton gli ardori, / era Teseo da dolce amor distretto, / in un giardin, pensando a suo diletto " (II, 3, p. 55). 
quotient in this docile creature, not even the stuff of a heroine of the medieval love story. " (p. 124) Le commentateur, tout en déniant à l'ceuvre de Boccace le caractère épique, reproche à Emilia sa fadeur et son effacement, et cela en reconnaissant quelques lignes plus loin que l'épopée ne laisse pas beaucoup de place aux protagonistes féminins : " In the great epics as in war itself the role of a woman is secondary or irrelevant 》 (p. 119). Malgré ces regards perplexes, la Teseida delle nozze d'Emilia offre aux lecteurs modernes un exemple éclairant de la conception médiévale et renaissante de l'art épique ; elle est le résultat de l'appropriation par Boccace des éléments épiques grecs et latins et d'une " refonte 》 de ceux-ci avec les références esthétiques de l'époque. Dès sa parution au milieu du $\mathrm{XIV}^{*}$ siècle, l'épopée de Boccace a servi de modèle aux poètes désireux d'illustrer leur langue maternelle en traitant le genre que les Anciens avaient réussi, surtout depuis Virgile, à marier à la notion de puissance politique. Bergin lui-même reconnaît cette facette de l'œuvre : " Not only for its manner but also for its matter, the Teseida was a seminal and influential work. If the reader of today finds it inflated, discursive, and not very persuasive, we must yet concede that the tale suited very well the tastes of its own times. " (p. 129)

Malgré l'apparente hardiesse des défenseurs de la langue vernaculaire, on ne saurait trop amplifier le choc épistémologique que représente une telle résolution. Boccace, une des figures dominantes dans l'esquisse, à cette époque, de l'illustration du vernaculaire, exprime néanmoins de la gêne et un malaise face à sa propre entreprise. Il écrit dans son Comento di Giovanni Boccaccio sopra la Commedia que le vulgaire toscan est efféminé en comparaison du latin, mais que le poème de Dante est néanmoins " orné » et " sublime "; et que Pétrarque, en vieillissant, est sorti de l'ombre de la langue vulgaire pour rejoindre les rangs des grands poètes grecs et latins (I, p. 85 et LVII, pp. 427-428). Plus il avança dans le chemin de sa vie, plus Boccace se repentit de ses années de " folies ». Il s'agit d'une situation particulière à l'Italie. Nous verrons bientôt que l'ascension du statut de la langue vernaculaire en Angleterre et en France s'effectua dans des conditions plus propices à l'épanouissement, sans aucune trace de pusillanimité ; 
mais il convient tout d'abord d'analyser, avec Pétrarque, cette disposition fort peu favorable à l'endroit de la langue vulgaire.

Quoique Pétrarque fât surtout célébré pour son quvre en langue vernaculaire toscane, le Canzoniere - qu'il appelait dédaigneusement Rerum vulgarium fragmenta -, il ne contribua pas à l'essor du projet d'illustration de la langue vulgaire. Bien au contraire: il ébrania les fondations encore très fragiles de l'entreprise. Selon les termes de Marc Fumaroli (1980), on s'évertua en effet, à partir de Pétrarque, à « [r]etrouver l'or pur de la latinitas enfoui dans le plomb de la décadence et de la barbarie " (p. 77). Il provoquait l'enthousiasme chez les latinistes qui en étaient à leurs premières grandes découvertes de manuscrits grecs et latins. En Italie, l'humanisme et l'entreprise d'illustration de la langue vulgaire se font une sérieuse concurrence. On peut même considérer, par exemple, les Elegantiarum linguae latinae libri de Lorenzo Valla émule de Pétrarque -, au début du XV' siècle, comme une sorte de " pied de nez " au projet de Dante. En effet, le dessein de Pétrarque n'était pas aussi solidement ancré que celui de Dante dans la conjoncture politique italienne. Il est vrai qu'en cherchant dans l'Antiquité latine la splendeur de la civilisation, il émettait en même temps un constat défavorable envers son époque', mais il ne proposa jamais plus qu'une esthétique de vie individuelle, comportant de nombreuses affinités avec celle des grands esprits de la culture grecque et latine. En fait, comme le remarque Alain Michel, en réconciliant la tradition des lettres antiques avec la production littéraire de son temps, il a transcendé les questions de

9. Nicholas Mann (1989) mentionne dans son Pétrarque, essai traduit de l'anglais par Édith McMorran et revu par l'auteur, que, " pour Pétrarque [...], toute la question de Rome et de l'Italie était un sujet fertile : ses vers italiens, certaines de ses églogues et de ses Invectlves, cuvres polémiques, regorgent de références aux beautés de son pays natal, à sa supériorité naturelle et culturelle sur la France, à l'état présent de désolation de Rome et à son potentiel comme capitale du monde » (p. 56). 
politique et s'est imposé comme l'emblème d'une « renaissance artistique ${ }^{10} \%$.

Ainsi Pétrarque ne montra pas beaucoup de tendresse pour la langue vulgaire, qui devint, de son point de vue, la langue grossière des " pestiférés 》. Dans une lettre familière adressée à son ami Boccace qui l'avait accusé de ne pas vouer suffisamment d'admiration à Dante, Pétrarque répond, cavalièrement, qu'en effet il ne s'intéresse que très peu à celui qui est si populaire auprès de la foule. Dans cette lettre, Boccace, en grand admirateur de Pétrarque, s'excuse et s'accuse, avec une touche d'ironie, d'avoir suivi durant sa jeunesse les traces de Dante. Pétrarque qui n'est pas dupe de ce mea culpa tente de désamorcer l'accusation de son ami, mais il ne fait que confirmer son mépris pour le Dante adulé, imité et reconnu par tous comme le plus grand poète italien - ce qui n'est pas sans connotation dépréciative pour Pétrarque. L'œuvre qui mena Pétrarque au sommet de la gloire dans son pays, le Canzoniere ou Fragments en langue vulgaire, ne fut jamais pour le grand latiniste qu'une source de regrets - bien qu'il puisse s'agir d'une forme amplifiée de la modestie affectée; la répétition, dans ses lettres familières, de ce sentiment de rejet par rapport à son œuvre en langue vulgaire, et ce à divers moments de sa vie, me semble significative. Il écrit, par exemple, dans cette lettre à Boccace :

Meque non frustra timuisse res indicat, quando in his ipsis paucis que michi iuveniliter per id tempus elapsa sunt, vulgi linguis assidue laceror, indignans quodque olim amaveram perosus; quotidie nolens et ingenio iratus meo in porticibus versor, ubique Dametas meus in triuijs solitus « stridenti miserum stipula disperdere carmen ». (Pétrarque, 1942, XXI, 15, p. 98)

[Et c'est pour cause que je pleurais, puisque je suis cruellement torturé par le carnage populaire de ces quelques poèmes de jeunesse que j'avais fait publier à l'époque, si bien que je suis dégoaté par ce que j'avais déjà aimé. [...] Je suis furieux contre

10. Cf. l'introduction qu'Alain Michel consacre à son Pétrarque et la pensée latine (1974). 
moi-même, car partout parmi les hordes d'illettrés je trouve mon propre Dametas qui, comme celui de Virgile, « massacre sur son instrument strident ma malheureuse chanson ${ }^{\prime \prime}$.]

Pétrarque posa un geste symbolique très significatif envers son « disciple », Boccace : fort conscient de l'incertitude et du malaise de celui-ci face à ses " ceuvres de jeunesse » en langue vernaculaire, Pétrarque lui fit l'《 honneur » de traduire en latin la dernière nouvelle de son Decameron.

Pétrarque, pionnier de la Renaissance, exalte en outre la perfectibilité de l'Homme et de l'individu. La gloire et le dépassement personnels acquièrent dans ses écrits une importance fondamentale. Toujours dans cette même lettre où il est surtout question de Dante, Pétrarque explique pourquoi, en tant que collectionneur de livres, il n'a pas cherché a se procurer le livre de Dante :

Eidem tunc stilo deditus, vulgari eloguio ingenium exercebam ; nichil rebar elegantius necdum altius aspirare didiceram, sed verebar ne si huius aut alterius dictis imbuerer, ut est etas illa flexibilis et miratrix omnium, vel invitus ac nesciens imitator evaderem. (Pétrarque, ibid, p. 96)

[J'ceuvrais alors dans la même veine, essayant mon éloquence en notre vulgaire. Je croyais que rien n'eût pu être plus splendide, car je n'avais pas encore appris à regarder plus haut. Mais je craignais qu'en me plongeant dans ses mots [...] je devinsse un imitateur, bien involontairement et sans meme m'en rendre compte.]

Et il adopte une attitude condescendante, lorsqu'il se défend de ressentir de l'envie face au grand poète :

Quam tandem veri faciem habet ut invideam illi qui in his etatem totam posuit, in quibus ego vix adolescentie florem primitiasque posuerim ? Ut quod illi artificium nescio an unicum, sed profecto

11. Les traductions du latin au français sont de nous. 
supremum fuit, michi iocus atque solatium fuerit et ingenii rudimentum ? (lbid, p. 98)

[Pourquoi aurais-je envié cet homme qui a consacré toute sa vie à ce genre d'ceuvres qui ne furent que les balbutiements, les premiers fruits de mon esprit ? Son cuvve, encore qu'elle ne fot pas sa seule creation, ne représente-t-elle pas certes sa plus grande rtalisation, tandis que pour moi ce n'était qu'un simple passe-temps, une consolation, le premier essai de mes talents ?]

La position téfractaire de Pétrarque dans le débat sur la langue vernaculaire est éclairante : elle nous indique d'abord, par le positionnement " subversif » de Pétrarque, l'existence d'une forte volonté au sein de la société « vulgaire » d'affirmer, de défendre et d'illustrer la langue maternelle italienne ; elle nous révèle aussi une certaine homogénéité esthétique dans la conception de certains genres poétiques, tels que l'épopée. En effet, malgré les divergences " idéologiques » entre les partisans d'une révolution linguistique et les tenants d'un retour complet à l'antiquité, on arrive, chez les poètes, à un certain consensus a propos du genre le plus noble, recommandé par Dante. Même Pétrarque qui prône une exacte conformité avec l'Antiquité n'exclut pas de son couve héroïque latine, Afric $^{12}$, les scènes amoureuses, interprétées comme des marques de dégénérescence par de nombreux commentateurs modernes. Son poème épique devait renfermer, explique Nicholas Mann (1989), " non seulement des scènes de batailles et de confrontations historiques, mais une histoire et une topographie de la Rome ancienne, des rêves prophétiques et une allégorie de la Vérité, un portrait de son protagoniste, et finalement une histoire d'amour inspirée par Didon et Énée, celle de Massinissa et de Sophonisbe " (pp. 70-71). Puisque nul n'est prophète en son pays, Dante, mais surtout Boccace, connurent en Angleterre d'abord, et en France ensuite, le succès qu'ils espéraient connaître en Italie : ils eurent le grand honneur de voir leurs ceuvres servir de modele aux

12. Notons au passage le sens symbolique de la résolution de Pétrarque de composer son cuvre illustre dans la langue de ses ancêtres romains. 
langues vernaculaires en plein essor. L'Italie, siège de l'Église, n'a pas suivi ce même mouvement de vulgarisation.

Avec Chaucer, le mouvement de " vulgarisation " prit de l'ampleur. En sortant de l'Italie, Dante et Boccace accédèrent au rang d'" auctoritates " en matière de langue vulgaire. Ils servirent de modèles aux poètes encore perplexes face à la possibilité de hisser leur vernaculaire au rang des grandes langues poétiques et philosophiques. Chaucer a largement contribué à la consécration de Boccace en tant que grand poète vernaculaire, en traduisant plusieurs de ses ceuvres italiennes et latines en son dialecte anglais. En adaptant au tout début de sa carrière poétique la Teseida delle nozze d'Emilia de Boccace, Chaucer a d'ailleurs conféré à cette ceuvre une valeur emblématique de l'entreprise d'illustration du vernaculaire.

Chaucer peut être reconnu comme l'un des "fondateurs " de la langue anglaise, en ce sens que son cuvre non seulement contribua à régler et à fixer l'orthographe et la syntaxe anglaises, mais encore encouragea et influença de nombreux poètes qui lui succédèrent. Si on ne peut réellement parler de " créateur" de la langue, on peut certainement évoquer son rôle. $d^{\prime}$ ' i illustrateur ${ }^{13}$ ". Chaucer est né avec le début de la Guerre de Cent ans, autour de 1340. Il a ressenti, dès son plus jeune âge, la menace de l'ennemi français, qui fut exacerbée par le roi Édouard III qui, pour susciter l'ardeur des patriotes, avivait sans cesse chez les Anglais la crainte d'être envahis et anéantis culturellement par les Français. Ce climat d'incertitude en rapport avec l'avenir de l'Angleterre donna lieu, d'ailleurs, à d'autres types d'entreprises linguistiques qui ne doivent pas être dissociées des conjonctures politiques. En effet, John

13. F. N. Robinson, dans son introduction aux CEuvres de Chaucer, The Works (1957), rappelle un commentaire du professeur Scherillo qui s'oppose aux commentateurs qui accordent trop aisément à Dante le rôle de "créateur » de la langue italienne et écrit : " A similar reminder might appropriately have been addressed to those writers who have called Chaucer the creator of English. Such a statement of course totally misrepresents the development of the language. " (p. xxx) 
Wycliffe (1320-1384) prêchait déjà, près de deux siècles avant Luther, un retour à l'interprétation personnelle de la Bible, qu'il entreprit lui-même de traduire en anglais. Chaucer, parfait exemple du poète-combattant, occupait lui aussi une position d'influence à la cour d'Angleterre ; il était écuyer et protégé du fils d'Édouard III, Jean de Gand, futur roi. On lui confia plusieurs missions diplomatiques en France et en Italie, qu'il accomplit avec une finesse remarquée par ses bienfaiteurs. Son travail d'écrivain fut prolifique et fascina pendant plusieurs siècles de nombreux poètes, tels que Spenser et Shakespeare. Parmi les cuvres d'une grande importance pour le développement du vernaculaire anglais, notons bien sûr les Canterbury Tales - d'où est tiré le " Knight's Tale » dont il sera question plus loin -, de même que Troilus and Criseyde et Anelida and Arcite.

Chaucer entreprit une de ses missions diplomatiques en Italie au début des années 1370 et put être témoin, à ce moment, du couronnement de la grande ceuvre de Dante, la Commedia, et de l'établissement d'une chaire en l'honneur de celle-ci. Ce qu'il découvrit lors de ce voyage est à l'origine de ses plus grandes réalisations. Il fut en mesure de recevoir de Dante l'inspiration qu'il recherchait pour la création d'une cuvre poétique en langue vernaculaire. En outre, il put se réjouir de connaître en son pays l'avantage fondamental, désigné par Dante pour mener à bien l'entreprise d'illustration du vulgaire : l'appartenance à une Cour royale pouvant régir l'usage de la langue (le vulgaire doit être ( cardinal, royal et courtois 》). Il n'eut ensuite qu'à considérer les autres recommandations de Dante (le choix entre les trois matières dignes du vulgaire illustre : prouesse d'armes, amour et vertu; la supériorité de la " chanson » par rapport à la ballade, au sonnet et à la prose ; et le choix du ton tragique, élégiaque ou comique) et à étudier de près la première version italienne « post-dantesque 》 de l'œuvre illustre - la Teseida delle nozze d'Emilia.

Les deux ceuvres de Chaucer qui présentent à mon avis le plus d'affinités avec le projet de Dante et de Boccace sont « The Knight's Tale " et Troilus and Criseyde, car elles contiennent des éléments épiques et, dans une certaine mesure, une grandeur tragique 
digne de l'cuvre la plus " illustre ». Le " geste " de traduction de la Teseida - " The Knight's Tale " - est porteur en soi d'un sens symbolique. Que Chaucer ait choisi d'en faire un «conte " de Canterbury au lieu d'une épopée n'enlève rien au projet tacite d'illustrer la langue maternelle des Anglais. D'ailleurs même si le caractère de cette cuuvre n'est pas purement épique, elle peut tout de même être qualifiée d' "illustre " pour cette raison que l'histoire nous est racontée par un chevalier courtois, au parler exquis ; Dante lui accorderait le mérite d'être cardinal, royal et courtois. En effet, on pourrait penser que Chaucer a voulu créer l'cuvre « totale ", en confondant sa traduction de la Teseida, emblème de l'œuvre vulgaire " illustre ", et le projet d'illustration à l'aide d'une autre ceuvre de Boccace, qui ressortit à un tout autre genre, à savoir le Decameron. Les « auditeurs » du " Knight's Tale » sont éblouis par la beauté de ce conte et considèrent sa valeur assez grande pour être conservée en mémoire :
Whan that the Knyght had thus his tale ytoold, In al the route nas ther yong ne oold That he ne seyde it was a noble storie, And worthy for to drawen to memorie ; And namely the gentils everichon. (Chaucer, in Robinson, ed., p. 47, vers 3109-3113)

Plusieurs critères me font rattacher cette ceuvre au projet de Dante. D'une part, ce récit est digne de la mention "illustre » par son inventio, puisqu'il s'agit de l'adaptation d'un texte qui se veut une épopée et que Chaucer en conserve tous les éléments essentiels (c'est-à-dire tout l'apparat mythologique, les combats, les jeux et les invocations aux dieux). D'autre part, l'" architecture" des Canterbury Tales suggère une hiérarchie entre chacun des récits : d'abord la localisation du " Knight's Tale » au tout début du recueil a pour effet de le mettre en évidence et d'inscrire les autres contes dans une dynamique de subordination. L'hôte de tous ces joyeux pèlerins, d'ailleurs, défie le prochain devisant d'égaler la performance du chevalier : 
[...] Lat se now who shal telle another tale ;

For trewely the game is wel bigonnee,

Now telleth ye, sir Monk, if that ye konne

Somwhat to quite with the Knyghtes taie. (Ibid, vers 3116-3119)

Ensuite, on peut souligner les dimensions disproportionnées du " Knight's Tale " par rapport aux autres contes, l'ampleur du récit lui conférant une autonomie qui pourrait permettre de le détacher de l'ensemble. Le "Knight's Tale " souscrit, tout en y apportant quelques variations, au projet d'abord dessiné par Dante, et concrétisé ensuite par Boccace.

Avec Troilus and Criseyde - cuvre composée environ au même moment que le "Knight's Tale " - Chaucer prend véritablement son envol épique. F.N. Robinson (1957), éđiteur des ceuvres de Chaucer, écrit à propos de Troilus : " The Troilus is Chaucer's supreme example of sustained narration, "The Knight's Tale" alone being in any way comparable. And it remains unsurpassed in its kind in later English poetry. " (p. 385) Dans ce récit, Chaucer fait appel à la muse épique, Calliope, afin d'assurer à son " livre " la beauté et la grandeur nécessaires. Dans les premiers vers du Livre III, le narrateur invoque la Muse de l'épopée :

Ye in my naked herte sentement inhielde, and do me shewe of thy swetnesse. -

Caliope, this vois be now present,

For now is nede, sestow nought my destresse[...]. (lbid., p. 421, vers 43-46)

Dans Troilus and Criseyde, Chaucer évoque constamment la présence de ses prédécesseurs immédiats, Dante et Boccace, en reprenant à son compte plusieurs formules de ces auteurs comme si les réalisations de ceux-ci n'avaient servi qu'à lui insuffler le désir d'illustrer sa langue maternelle. Par exemple, au début du Livre IV, le narrateur s'adresse à son livre, tout comme l'avait fait Boccace dans la Teseida et comme Dante le suggérait dans le Convivio ( $\mathrm{Je}$ me tourne vers la chanson elle-même, sous le visage de mon propre discours, et parle à icelle ", Banquet, p. 343) et lui souhaite de faire 
bonne route et de suivre les traces des plus grands " poèmes 》, en évoquant les cinq mêmes poètes que Dante avait aussi implorés au début de l'Enfer :

Go, litel bok, litel myn tragedye,

Ther God thi makere yet, er that he dye,

So sende myght to make in som comedye!

But litel book, no makyng thow n'envie,

But subgit be to all poesye ;

And kis the steppes, where as thow seest pace

Virgile, Ovide, Omer, Lucan, and Stace. (lbid, p. 479, vers 17861792)

En France, le projet de Dante eut aussi des répercussions mais ta connivence entre les auteurs n'est pas aussi évidente et se laisse moins bien saisir que dans les textes de Chaucer, par exemple. Déjà avec Nicole Oresme, vers 1370 , il est question, par la translatio studii, d'un transfert du savoir du latin au français (Lusignan, 1986, p. 154). Oresme participe-t-il aussi de ce même projet? Dans l'affirmative, son ceuvre n'aurait pas contribué à rendre illustre la langue vernaculaire, mais plutôt à orienter le débat et la réflexion vers les enjeux politiques d'une « illustration 》 de la langue française.

De son côté, Alain Chartier fait figure de jalon dans l'ébauche d'un portrait historique de la langue française. On le constate, me semble-t-il, à la lecture de quelques-uns de ses textes, parce qu'au premier objectif, celui de la translatio studii, le passage, la translation d'une langue en une autre, il en ajoute un autre qui accentue davantage les conséquences politiques favorables : l'idée de la " patrie » forte et unie. Les ceuvres politiques les plus connues d'Alain Chartier, le Quadrilogue invectif, le De detestatione belli gallici et suasione pacis, le Lay de Paix révèlent un auteur que l'on pourrait qualifier de "patriotique ». Dans le Quadrilogue invectif (1422), Chartier fait appel au courage et à la fierté des Français qui se trouvaient alors dans une situation politique précaire; il tente de provoquer et d'organiser une résistance contre l'Angleterre afin de préserver l'unité de sa patrie. On peut lire au début de l'ouvrage : 
Et le peuple appele Scite, en la guerre qu'il eut avecques le roy Daire de Perse, se mist toujours en fuyte jusques ad ce qu'il vint au liew ou estojent les sepulhures de leurs peres et predecesseurs, et illec se combattirent jusques a la mort, come ceulx qui pitie naturelle de leurs parents et payz contrajgnoit a resistence et a garder le lieu de la naissance et sepulture de leurs lignees. (Chartier, 1923, p. 11)

Ce " pamphlet " eut un tel succès qu'on le traduisit et le publia en Angleterre. En effet, la situation incertaine des Français autour des années 1430 correspondant aux conjonctures politiques anglaises de la deuxième moitié du $\mathrm{XV}^{\circ}$ siècle, les propos de Chartier parvinrent aussi à rejoindre la sensibilité « patriotique » des Anglais !

Chartier a illustré la langue frangaise dans le genre lyrique. La Belle dame sans mercy (1424) fut reconnue comme l'une des grandes ceuvres de l'époque. Son succès fut tel que l'on peut aujourd'hui parler d'un "cycle " de la Belle dame sans mercy". En effet, on l'imita tellement et si bien que, un siécle plus tard, on considérait toujours Alain Chartier comme un maître de l'éloquence. Dans ce poème lyrique composé de strophes en vers octosyllabiques, on ne retrouve aucune allusion linguistique ou politique, telle que l'on pouvait en découvrir dans l'ceuvre de Chaucer. Pour cette raison on pourrait croire que ses compositions ne sont que purs " divertissements » courtois, mais il me semble peu probable qu'un " poète-combattant " tel Alain Chartier, qui de surcroît connaissait bien les grands auteurs italiens, ait écrit dans l'unique but d'être " plaisant ». En situant Chartier dans la continuation du " débat " inspiré par Dante, Piaget répondrait lui-même à l'interrogation qu'il formule dans l'introduction de son édition de la Belle dame sans mercy : " Comment maître Alain, secrétaire du roi et archidiacre de Paris, pouvait-il, oubliant tout cela [la situation politique précaire], se lamenter sur la mort de sa dame et composer, à l'usage des galants, un poème rempli de fadaises amoureuses ? $»$ (p. viii) Je crois tout à fait pertinent de lier cette poesie qui, selon Piaget,

14. Voir l'intróduction d'Arthur Piaget (p. 12) de l'édition Droz (1949). 
manquerait « assurément d'imagination réelle et de toute vérité de sentiments 》 (p. ix) à l'entreprise d'illustration dans le genre amoureux, une des trois matiêres préconisées par Dante. La Belle dame sans mercy développe, à la manière du Canzoniere, déjà célèbre à l'époque, des thèmes typiques du genre lyrique médiéval.

Une première traduction française de la Teseida de Boccace parut vers 1460 sous le titre de Thezeo. Cette traduction étant conservée par de nombreux manuscrits, il est permis d'affirmer que l'œuvre connut un réel succès, mais peu de documents attestent par ailleurs de son existence et de sa diffusion. Le contexte politique de la deuxième moitié du XV' siècle, comme nous l'avons déjà observé, semble favoriser la traduction d'une ceuvre dont la forme et la matière convient de nouveaux adeptes à un rassemblement idéologique. Les " dames de cour 》, pour qui la Teseida fut traduite, s'il faut en croire les préfaces, devaient participer, elles aussi, au grand déploiement d'énergies visant à imposer la langue " aulique » à tout le royaume de France. Le récit " épique » offre, d'une part, l'avantage de la polysémie métaphorique - combat guerrier et combat linguistique pouvant être placés sur le même niveau sémantique, " la plume et la langue des orateurs comme les glaives des combattans " - et, d'autre part, la possibilité d'élaborer de grandes descriptions romanesques, fort utiles au développement de la langue, et, dit-on, grandement appréciées des dames.

Claude de Seyssel prit le relais au tournant du siècle. Secrétaire et traducteur du roi, il exhorta, à son tour, les poètes à " enrichir et magnifier " leur langue ${ }^{15}$. Il délégua donc cette tâche qui semble, par le ton de son discours, d'une valeur capitale, à d'autres écrivains que lui-même, en espérant - on l'imagine - que les répliques seraient enthousiastes. Il me faut rappeler ici un détail important que relève Ferdinand Brunot : cette requête auprès des poètes français qui apparaissait dans la préface d'une traduction de l'Histoire de Justin ne fut pas publiée avant 1559 . Toutefois, il ne

15. Cf. Ferdinand Brunot (1894, pp. 27-37, et 1906, tome II, livre I, chap. 3). 
faudrait pas croire pour autant que cette traduction n'ait pas circulé. Cette ceuvre fut traduite en français afin que le roi et son entourage profitent des enseignements moraux de l'auteur ; elle a donc pu connaître une certaine diffusion à la Cour. Cette cuvre, qu'elle ait rencontré ou non des lecteurs, reflète une atmosphère politique toute particulière qu'il convient d'associer à un projet linguistique que les Italiens connaissaient déjà depuis le XIV' siècle.

Anne de Graville appartient à la même époque. Grande admiratrice d'Alain Chartier, elle composa d'abord - sans doute entre tes années 1515 et 1520 - des rondeaux s'inspirant de la Belle dame sans mercy ${ }^{16}$. Le succès qu'elle remporta avec ce premier essai poétique lui valut peut-être l'honneur de traduire " pour la Reine " une cruvre qui avait déjà connu énormément de succès dans sa version de 1460 - le Thezeo. La reine Claude lui montrait d'ailleurs beaucoup d'estime et de confiance en tui demandant d'embellir et de « rajeunir », en 1521, un texte italien existant déjà en version française. Il faut croire qu'elle s'acquitta de sa tâche avec brio, puisque, on se le rappellera, at moins cinq manuscrits du Beau romant des deux amans et de la belle et saige Emilia circulèrent en son temps.

Anne de Graville - aussi connue sous le nom de « Ma Dame Dentragues $"$ - translata le Thezeo de 1460 en un français plus " moderne » et plus particulier à la Cour de la reine Claude. Les modifications qu'elle apporta au Thezeo étaient conformes aux recommandations de Dante. En tant que membre privilégié de la Cour royale, elle assurait, tout comme son prédécesseur français, au vulgaire illustre un attribut essentiel : un brillant « cardinal, royal et courtois 》. Anne de Graville remania le roman en prose qu'elle avait entre les mains en une " chanson » qui, grâce au vers héroique, possédait une résonnance épique. Tout comme Chaucer, elle ne sut se restreindre à choisir une seule des " matières illustres " prouesse d'armes, amour et vertu -, si bien que son cuvre reflète

16. Ces rondeaux, publiés par Walhund en 1897, se trouvent à la BN de Paris, man. fr. 2253. 
plutôt un désir d'incorporer dans un seul livre toutes les dimensions de la poésie ; et cette manière ancrée dans l'esthétique médiévale ne se définit pas en opposition avec l'épopée classique; elle se situe plutôt dans sa continuité.

La renommée d'Anne de Graville était si grande en France qu'au $\mathrm{XIX}^{\complement}$ siècle on retrouve encore son nom dans les registres officiels, entre autres dans le Dictionnaire historique, littéraire et bibliographique dés Françaises de Fortunée Briquet $(1804)^{17}$. Ses contemporains la connaissaient comme femme de lettres et la citaient parfois comme exemple d'éloquence. En effet, Geofroy Tory (1970), auteur et imprimeur à Paris, cita un des rondeaux de la Dame Dentragues pour « monstrer que nostre dict langage Francois a grace quant il est bien ordonné " (fol. iv). Dans le traité intitule Champ fleury, il exprime son désir de régler la langue française :

S'it est vray que toutes choses ont eu commancement, il est certain que la langue Grecque, semblablement la Latine ont este quelque temps incultes \& sans Reigle de Grammaire, comme est de present la nostre, mais les bons Anciens vertueux \& studieux ont prins peine, \& mis diligence a les reduyre \& mettre a certaine Reigle, pour en user honnestement, a escripre $\&$ rediger les bonnes Sciences en memoire, au prouffit $\&$ honneur du bien public. (Ibid, fol. iv, verso)

Reconnu comme l'un des premiers à publier un projet d'illustration de la langue française, Geofroy Tory, tout comme ses prédécesseurs, de Dante à Anne de Graville, défendit une esthétique de cour que la Pléiade allait dénigrer quelques années plus tard.

Si l'histoire littéraire a surtout retenu la Défense et illustration de Joachim Du Bellay, c'est que celui-ci et le groupe dont il était le porte-parole se sont eux-mêmes affirmés comme les premiers " vrais " poètes de la langue française. "Comme un sergent de bande de la langue française ", Du Bellay (1948, p. 98)

17. L'historienne Suzanne Pellegrin (CNRS) se propose de réediter le Dictionnaire de Fortunée Briquet. 
a mis plusieurs poètes-combattants « mal armés » hors de combat. Les poètes, tels Alain Chartier et Anne de Graville, ayant ceuvré à l'illustration de la langue française avant la Pléiade furent désignés par Du Bellay comme des " mal équipés » qui auraient eu avantage à se retirer " sous les frais ombrages, aux somptueux palais des grands seigneurs et cours magnifiques des princes, entre les dames et damoiselles [...] 》 (p. 255). En 1578, Henri Estienne (1980) qualifia l'esthétique poétique de ces poètes de $\operatorname{cour}^{18}$ de " courtisanisme ", terme dont Marc Fumaroli (1981) s'est inspiré pour désigner, à son tour, le mouvement opposé à cette esthétique, l'《 anti-aulicisme » (p. 141).

En imitant le modèle du De vulgari eloquentia (qui donna lieu à l'adaptation de Speroni, le Dialogo delle lingue, en 1542), Du Bellay se ralliait pourtant au rival de Dante, Pétrarque. La forme que Dante qualifiait d'inférieure au rondeau et à la ballade, le sonnet (illustré par Pétrarque), devient, pour les poètes de la Pléiade, une forme privilégiée. Le discours littéraire des poètes de la Pléiade est en accord avec celui des Anciens. Leur réflexion est alimentée par celles de poètes comme Horace, mais aussi de grands orateurs comme Cicéron ef Quintilien, tandis que l'esthétique littéraire des poètes de cour, de Chartier à Marot, s'inspira certes des Anciens, mais elle s'inscrit davantage dans une tradition établie par leurs prédécesseurs français et italiens. Il n'est donc pas étonnant que, lors d'une intense époque d'humanisme, on ait dénigré une poétique qui ne faisait pas suffisamment de place aux idées et aux préceptes des Anciens. Il ne faut pas s'étonner, non plus, du fait qu'on ait " oublié ", au siècle suivant, de rendre hommage à ces poètes de l'« enfance " de la langue française, car, comme l'écrit François Paré (1994), « les discours dominants n'ont pas de commencement

18. Cette expression, " poètes de cour \#, n'a de sens qu'à partir du moment où l'on définit une esthétique propre à ce groupe. À la lecture de la Défense et illustration de Joachim Du Bellay et de la " Préface sur la Franciade " de Ronsard, il est possible d'élaborer une esthétique littéraire qui diffère considérablement de celle des poètes que Du Bellay et Ronsard qualifiaient de « courtizan ". 
dans le raconté 》 (p. 38). La " fraternité » linguistique des débuts de l'évolution de la langue française, c'est-à-dire la volonté d'intégrer tous les parlers régionaux dans le grand projet d'unification de la langue, se transforme, dans la dernière phase de l'entreprise, en hostilité envers ]'Autre et en refus de celui-ci : « [l]e rassemblement ne sert plus à accueillir, à multiplier l'intervention dans le social et le politique, il sert à présider, juger, condamner, exclure " (p. 113).

\section{Universite McGill}

\section{Références}

BERGIN, Thomas G. (1981). " The Teseida of the Nuptials of Emilia », Boccaccio. New York, Viking Press.

BOCCACE (1888), Vita di Dante. Florence, Sansoni Editore.

(1895). Il Comento di Giovanni Boccaccio sopra la Commedia. Florence, Le Monnier.

(1992). Teseida delle nozze d'Emilia. Milan, Arnoldo Mondadori Editore.

BRIQUET, Fortunée (1804). Dictionnaire historique, littéraire et bibliographique des Françaises et des étrangères naturalisées en France, connues par leurs écrits, ou par la protection qu'elles ont accordée aux gens de lettres, depuis l'établissement de la monarchie jusqu'à nos jours. Paris, Imprimerie de Gille.

BRUNOT, Ferdinand (1894). " Un Projet d'"enrichir, magnifier et publier" la langue française en 1509 ", Revue d'histoire littéraire de la France.

(1906). Histoire de la langue française des origines à 1900 . Paris, Armand Colin. 
CASTIGLIONE (1991). Le Livre du courtisan, trad. par Alain Pons. Paris, Flammarion-GF.

CHARTIER, Alain (1923). Le Quadrilogue invectif. Genève, Droz.

CHAUCER (1957). " The Knight's Tale » in F. N. Robinson, dir. (1957).

DANTE (1965). "De l'éloquence en langue vulgaire ", Gurvres complètes, trad. André Pézard. Paris, Gallimard, La Pléiade.

DEMONET, Marie-Luce (1992). Les Voix du signe : nature et origine du langage à la Renaissance, 1480-1580. Paris, Honoré Champion.

ESTIENNE, Henri (1980). Deux dialogues du nouveau langage françois, éd. par P. M. Smith. Genève, Slatkine.

FUMAROLI, Marc (1980). L'Âge de l'éloquence. Rhétorique et "res literaria " de la Renaissance au seuil de l'époque classique. Genève, Droz.

(198.1). « Aula arcana. Rhétorique et politique à la cour de France sous Henri III et Henri IV ", Journal des savants, avril-juin.

MANN, Nicholas (1989). Pétrarque, trad. par Edith McMorran. Arles, Actes Sud, « Babel ».

MICHEL, Alain (1974). Pétrarque et la pensée latine. tradition et novation en littérature. Avignon, Aubanel.

LUSIGNAN, Serge (1986). Parler vulgairement : les intellectuels et la langue française aux XIIF et XIV siècles. Paris, Vrin.

PARÉ, François (1994). Les Littératures de l'exiguité. Ottawa, Le Nordir.

PÉTRARQUE (1942). Familiari. Florence, Sansoni Editore. 
ROBINSON, F. N., dir. (1957). Chaucer - The Works. Boston, Houghton Mifflin.

TORY, Geofroy (1970). Champ fleury, introduction de J. W. Joliffe. New York, Johnson Reprint, Mouton éd.

RÉSUMÉ : Les Projets d'« illustration » de la langue vernaculaire et leurs héritages littéraires - Dante écrivit, peu après Vita nova, en 1305, son De Vulgari eloquentia, texte qui servit de " manifeste ", d'abord aux champions des vernaculaires italiens, ensuite aux défenseurs des langues vulgaires européennes en pleine expansion dès la fin du XIV ${ }^{\circ}$ siècle. Dante suggérait aux poètes désireux d'" illustrer " leur langue maternelle de composer une ceuvre aussi magnifique que celle de son grand maître romain, Virgile. Une cuvre sans dénomination générique, mais définie par son vers hendécasyllabe et ses matières "illustres " : salus, virtus, venus. Boccace fut le premier disciple de Dante. Il répondit à l'appel de son maître, en écrivant une ceuvre dédiée à Mars, le tout-puissant dieu guerrier, seul capable d'accorder la gloire éternelle aux pauvres mortels. La Teseida delle nozze d'Emilia servit de modèle, quelques années seulement après sa parution, aux poètes anglais et français. Chaucer traduisit la Teseida, qui devint « The Knight's Tale ", et l'intégra à ses Canterbury Tales vers 1380 . En France, un traducteur anonyme du roi René d'Anjou traduisit à son tour, vers 1460 , l'œuvre épique de Boccace, qu'il intitula Thezeo. Au XVI' siècle, époque déterminante dans l'histoire de la langue française, Anne de Graville « translata de vieil langage en nouveau » le Thezeo qu'elle intitula: le Beau romant des deux amans Palamon et Arcita et de la belle et saige Emilia. Cette étude suit le parcours de la Teseida de Boccace d'une langue à une autre, en s'intéressant au projet et au " contre-mouvement" de l'illustration des langues vernaculaires européennes esquissé par le grand poète toscan, Dante.

ABSTRACT : Projects to 'illustrate' Vernacular Language and Their Literary Heritage - In 1305, shortly after his Vita nova, Dante wrote his De vulgari eloquentia, a text which served as a 'manifesto' for the champions of the Italian vernaculars and for the 
defenders of the European vulgar tongues which were rapidly expanding at the end of the 14th century. Dante suggested that poets wanting to 'illustrate' the mother tongue should compose a work as magnificent as that of his great Roman master Virgil, a work not defined generically but by its eleven-syllable metre and its 'illustrious' subject: salus, virtus, venus. Boccaccio, the first of Dante's disciples, responded to Dante's call by writing a work dedicated to Mars, the all-powerful warrior god who alone could grant eternal glory to poor mortals. Only a few years after it appeared, the Teseida della nozze d'Emilia served as a model for English and French poets. Chaucer translated the Teseida, which became "The Knight's Tale," and, around 1380, included it in his Canterbury Tales. In France, an anonymous translator at the court of King René of Anjou translated Boccaccio's text around 1460, giving it the title Thezeo. In the 16th century, a period of fundamental importance in the history of the French language, Anne de Graville translated the Thezeo into more modern French and gave it the title: Le Beau romant des deix amans Palamon et Arcita et de la belle et saige Emilia. Our study follows Boccaccio's Teseida from one language to another, taking particular interest in Dante's project, and in the 'counter movement,' to illustrate the European vernaculars.

Mawy Bouchard: Département de langue et littérature françaises, Universtté McGill, 3460 rue McTavish, Montréal (Québec) H3A 1 X9 Canada. 